\title{
ON THE DIOPHANTINE INEQUALITY
}

$$
\left|X^{2}-c X Y^{2}+Y^{4}\right| \leq c+2
$$

\section{Bo He, István Pink, Ákos Pintér and Alain Togbé}

ABa Teacher's College, P. R. China, University of Debrecen, Hungary, Hungarian Academy of Sciences and University of Debrecen, Hungary and

Purdue University North Central, USA

\footnotetext{
Abstract. Generalizing some earlier results, we find all the coprime integer solutions of the Diophantine inequality

$$
\left|X^{2}-c X Y^{2}+Y^{4}\right| \leq c+2, \quad(X, Y)=1,
$$

except when $c \equiv 2(\bmod 4)$, in which case we bound the number of integer solutions. Our work is based on the results on the Diophantine equation

$$
A X^{4}-B Y^{2}=C,
$$

where $A, B$ are positive integers and $C \in \pm\{1,2,4\}$.
}

\section{INTRODUCTION}

In recent years, several results have been established concerning solutions of quartic Thue equations and inequalities, see $[10,16,17]$ and the references given there. Let $c$ be a positive integer. In [7], Cusick resolved an infinite family of Diophantine equations $X^{4}-c X^{2} Y^{2}+Y^{4}=1$ and this result was generalized by Cohn [6] and Walsh [21] to the equations $X^{2}-c X Y^{2}+Y^{4}=$ $\pm 1, \pm 2, \pm 4$, cf. Lemma 2.1. Further, here we would like to mention two other theorems related to the quartic Thue inequalities. In 1997, Wakabayashi $[20$, Theorem 2] proved that the only primitive solutions (i. e. $(X, Y)=1)$ of the Thue inequality

$$
\left|X^{4}-a^{2} X^{2} Y^{2}+Y^{4}\right| \leq a^{2}-2 \quad(a \geq 8)
$$

2010 Mathematics Subject Classification. 11D25, 11J86.

Key words and phrases. Diophantine equations, quartic equations. 
are $(X, Y)=(0,0),( \pm 1,0),(0, \pm 1),( \pm a, \pm 1),( \pm 1, \pm a),( \pm 1, \pm 1)$ with mixed signs. Dujella, Ibrahimpašić and Jadrijević [8] resolved the Thue inequality

$$
\left|X^{4}-2\left(n^{2}-1\right) X^{2} Y^{2}+Y^{4}\right| \leq 2 n+3,
$$

where $n \geq 0$ is an integer.

As a common generalization of the previous inequalities, let us consider the Diophantine equation

$$
X^{2}-c X Y^{2}+Y^{4}=\mu, \quad|\mu| \leq c+2 .
$$

Determining completely the integer solutions of equation (1.1) depends on a conjecture on the number of solutions to Diophantine equation

$$
a X^{4}-b Y^{2}=1 .
$$

In 2005, Togbé, Voutier and Walsh [18] made the following conjecture.

Conjecture 1.1. Let $t>1$ denote a positive integer. Then the only positive integer solution to

$$
(t+1) X^{4}-t Y^{2}=1
$$

is $(X, Y)=(1,1)$, unless $t=m^{2}+m$ for some positive integer $m$, in which case there is also the solution $(X, Y)=\left(2 m+1,4 m^{2}+4 m+3\right)$.

We note that using the function SIntegralLjunggrenPoints in the computer algebra program package MAGMA [5] that the conjecture above is true for $1<t \leq 1000$ apart from the possible values of

$$
t \in\{388,502,634,702,744,772,820,829,878,883,953,955,956,957,993\} .
$$

In these exceptional cases MAGMA failed to find all integer points on the quatric, because it was not able to compute the rank of the corresponding elliptic curve.

Our main result is the following.

TheOREM 1.2. For a positive integer $c \geq 3$, all of the coprime integer solutions $(X, Y)$ of Diophantine equation (1.1) are given by

(1.3) $(X, \pm Y)=(-1,0),(1,0),(0,1),(-1,1),(1,1),(c-1,1),(c, 1),(c+1,1)$, expect for the following cases:

1. If $c=338$, then equation (1.1) has the additional integer solution $(X, \pm Y)=(114243,6214),(13051348805,6214)$.

2. If $c=n^{2}$, then equation (1.1) has the additional integer solution $(X, \pm Y)=(1, n),\left(n^{4}-1, n\right)$.

3. If $c=n^{2}-1$, then equation (1.1) has the additional integer solution $(X, \pm Y)=(1, n),\left(n^{4}-n^{2}-1, n\right)$.

4. If $c=n^{2}+1$, then then equation (1.1) has the additional integer solution $(X, \pm Y)=(1, n),\left(n^{4}+n^{2}-1, n\right)$. 
5. $c \equiv 2(\bmod 4)$ is not as in $(1.2)$, with $\mu=-c+2$. In this exceptional case, there are at most two additional solutions $(X, Y)$ to equation (1.1). Under Conjecture 1.1, there is no other additional solution.

For $c=1$ and 2 we can give all the solutions by using elementary considerations. Indeed, if $c=2$ then we have

$$
x^{2}-2 x y^{2}+y^{4}=\left(x-y^{2}\right)^{2} \leq 4
$$

and $x-y^{2}=0, \pm 1, \pm 2$, so all the solutions are

$$
(x, y)=\left(m^{2}, m\right),\left(m^{2} \pm 1, m\right),\left(m^{2} \pm 2, m\right),
$$

where $m$ is an integer. For $c=1$ we obtain

$$
\left|x^{2}-x y^{2}+y^{4}\right|=\frac{x^{2}+\left(x-y^{2}\right)^{2}+y^{4}}{2} \leq 3,
$$

and a straightforward calculation shows that all the solutions are

$$
(x, y)=( \pm 1, \pm 1),(-1,0),(1,0),(0, \pm 1),(0,0),(2, \pm 1) .
$$

The organization of this paper is as follows. In Section 2, we will recall some useful results, particularly results related to the Diophantine equation

$$
A X^{4}-B Y^{2}=C,
$$

where $A, B$ are positive integers and $C \in \pm\{1,2,4\}$. The proof of Theorem 1.2 will be done in Section 3 by the means of the results cited in Section 2 .

\section{Preliminaries}

In 1995, Cohn [6] considered the Diophantine equation

$$
x^{2}-c x y^{2}+y^{4}=1,
$$

where $c$ is an odd positive integer. Walsh [21] solved the equation with $c$ an even positive integer.

Lemma 2.1 (Cohn 1995, Walsh 1999). The only solutions to $x^{2}-c x y^{2}+$ $y^{4}=1$ in non-negative integers $(x, y)$ are $(c, 1),(1,0),(0,1)$, unless either $c$ is a perfect square, in which case there are also the solutions $(1, \sqrt{c}),\left(c^{2}-\right.$ $1, \sqrt{c})$, or $c=338$ in which there are the solutions $(x, y)=(114243,6214)$, $(13051348805,6214)$.

We will also recall some classical results on the quartic Diophantine equation

$$
a X^{2}-b Y^{4}= \pm 1, \quad \pm 2, \quad \pm 4 .
$$

We will consider the positive values of the constants in equation (2.1) and recall two results.

The result on the Diophantine equation

$$
a X^{2}-b Y^{4}=4, \quad 2 \nmid a b,
$$


was obtained by Luo and Yuan [15]. Let $\left(x_{1}, y_{1}\right)$ be the minimal positive integer solution of equation $a x^{2}-b y^{2}=4$. We define $x_{k}, y_{k}$ by

$$
\left(\frac{x_{1} \sqrt{a}+y_{1} \sqrt{b}}{2}\right)^{k}=\frac{x_{k} \sqrt{a}+y_{k} \sqrt{b}}{2}, \quad 2 \nmid k .
$$

Lemma 2.2 (Luo and Yuan, 2007). 1. If $y_{1}$ is not a square, then the equation (2.2) has no positive integer solution expect for the case $y_{1}=$ $3 \square$ and by $y_{1}^{2}+3=3 \square$, when $(X, Y)=\left(x_{3}, \sqrt{y_{3}}\right)$ is the only solution of (2.2).

2. if $y_{1}$ is a square, then equation (2.2) has at most one positive integer solution other than $(X, Y)=\left(x_{1}, \sqrt{y_{1}}\right)$, which is either $(X, Y)=$ $\left(x_{3}, \sqrt{y_{3}}\right)$ or $(X, Y)=\left(x_{2}, \sqrt{y_{2}}\right)$, the latter occurring if and only if $x_{1}$ and $y_{1}$ are both squares and $a=1, b \neq 5$.

For the Diophantine equation

$$
a X^{2}-b Y^{4}=2, \quad 2 \nmid a b,
$$

Ljunggren [12] proved that the equation has at most two solutions in positive integers $(X, Y)$. Luca and Walsh [14] obtained a more precise result. In fact, let $\left(u_{1}, v_{1}\right)$ be the minimal positive integer solution of equation $a x^{2}-b y^{2}=2$. We also define $u_{k}, v_{k}$ by

$$
\left(\frac{u_{1} \sqrt{a}+v_{1} \sqrt{b}}{\sqrt{2}}\right)^{k}=\frac{u_{k} \sqrt{a}+v_{k} \sqrt{b}}{\sqrt{2}}, \quad 2 \nmid k .
$$

LEMma 2.3 (Luca and Walsh, 2001). 1. If $v_{1}$ is not a square, then equation (2.4) has no solutions.

2. If $v_{1}$ is a square and $v_{3}$ is not a square, then $(X, Y)=\left(u_{1}, \sqrt{v_{1}}\right)$ is the only solution of equation (2.4).

3. If $v_{1}$ and $v_{3}$ are both squares, then $(X, Y)=\left(u_{1}, \sqrt{v_{1}}\right)$ and $\left(u_{3}, \sqrt{v_{3}}\right)$ are the only solutions of equation (2.4).

We recall here another result obtained Ljunggren [12].

Lemma 2.4 (Ljunggren, 1954). The Diophantine equation $a X^{2}-b Y^{4}=1$ has at most one positive integer solution $(X, Y)$ with $a>1$.

For the negative values of the constants in equation (2.1), first we consider the equation

$$
a X^{4}-b Y^{2}=4 .
$$

We proceed like for Lemma 2.2 by considering the definition of $x_{1}, y_{1}$ and that of of $x_{k}, y_{k}$ given by equation (2.3). Ljunggren [13] proved the following result.

Lemma 2.5 (Ljunggren, 1967). If $2 \nmid a b$, then the Diophantine equation $a X^{4}-b Y^{2}=4$ has at most two positive integer solutions $(X, Y)$ with $2 \nmid X Y$. 
1. If $x_{1}=h^{2}$ and $a x_{1}^{2}-3=s^{2}$, there are only two solutions, namely, $(X, Y)=\left(\sqrt{x_{1}}, y_{1}\right),\left(\sqrt{x_{3}}, y_{3}\right)$.

2. If $x_{1}=h^{2}$ and $a x_{1}^{2}-3 \neq s^{2}$, then $(X, Y)=\left(\sqrt{x_{1}}, y_{1}\right)$ is the only solution.

3. If $x_{1}=5 h^{2}$ and $a^{2} x_{1}^{4}-5 a x_{1}^{2}+5=5 s^{2}$, then the only solution is $(X, Y)=\left(\sqrt{x_{5}}, y_{5}\right)$.

In 2009, Yuan and Li [22] confirmed a conjecture posed by Akhtari, Togbé and Walsh $[2,3]$. See also [19].

Lemma 2.6 (Yuan and Li, 2009). If $2 \nmid a b$, then the Diophantine equation $a X^{4}-b Y^{2}=2$ has at most one positive integer solution $(X, Y)$.

The most difficult equation in (2.1) is

$$
a X^{4}-b Y^{2}=1 .
$$

The upper bound of the number of solutions to equation (2.6) is obtained by Akhtari [1] and Yuan-Zhang [23], independently. They showed the following result.

Lemma 2.7 (Akhtari 2009, Yuan and Zhang 2010). The Diophantine equation (2.6) has at most two positive integer solutions $(X, Y)$. [4].

A special case of the equation was studied by Bennettt, Togbé and Walsh

Lemma 2.8 (Bennett, Togbé, and Walsh, 2006). Let $m$ be a positive integer. Then the only positive integral solutions to the equation

$$
\left(m^{2}+m+1\right) X^{4}-\left(m^{2}+m\right) Y^{2}=1
$$

are given by $(X, Y)=(1,1)$ and $(X, Y)=\left(2 m+1,4 m^{2}+4 m+3\right)$.

\section{Proof of Theorem 1.2}

If one of the variables $X$ and $Y$ is zero, under the condition $(X, Y)=1$, then the other variable is \pm 1 . So we will assume that $X Y \neq 0$.

From (1.1) we have

$$
\left(2 X-c Y^{2}\right)^{2}-\left(c^{2}-4\right) Y^{4}=4 \mu
$$

So we will consider the equation

$$
x^{2}-\left(c^{2}-4\right) y^{2}=4 \mu, \quad|\mu| \leq c+2,
$$

where $x=\left|2 X-c Y^{2}\right|, y=Y^{2}$.

Using the same argument as in [9, Section 3], if equation (3.1) has a solution $(x, y)$, then we have

$$
\mu=1,-c+2 \text { or } c+2 .
$$


3.1. The case $\mu=1$. By the work of Cohn and Walsh, stated as Lemma 2.1 , the only solutions are $(X, \pm Y)=(0,1),(1,0),(c, 1)$, and the exceptional solutions in the cases (1.2) and (1.2) of Theorem 1.2.

3.2. The case $\mu=c+2$. From the equation

$$
x^{2}-\left(c^{2}-4\right) y^{2}=4(c+2) \text {, }
$$

we see that $(c+2) \mid x^{2}$. Put $c+2=g h^{2}$ with $g$ square-free. Then we have $x=g h z$ with integers $g, h$, and $z$. Therefore, the above equation can be rewritten into

$$
g z^{2}-(c-2) y^{2}=4
$$

One can see that the minimal solution is $\left(z, y_{1}\right)=(h, 1)$. By some results on Pellian equations, for any possible common divisor of $g z$ and $(c-2) y$, all of positive integer solutions to equation (3.2) are given by

$$
\frac{z \sqrt{g}+y \sqrt{c-2}}{2}=\left(\frac{z_{1} \sqrt{g}+y_{1} \sqrt{c-2}}{2}\right)^{k}=\frac{z_{k} \sqrt{g}+y_{k} \sqrt{c-2}}{2}, \quad 2 \nmid k .
$$

Using the above equality or classical properties of Lucas-Lehmer numbers [11], we have $z_{1} \mid z_{k}$, i.e. $h \mid z$. Thus, equation (3.2) becomes

$$
(c+2) x^{2}-(c-2) y^{2}=4 .
$$

Combining this and equation (3.1), we will study the equation

$$
(c+2) x^{2}-(c-2) Y^{4}=4,
$$

where $x^{\prime}=\frac{\left|2 X-c Y^{2}\right|}{c+2}$.

It is easy to see that $\left(x^{\prime}, \pm Y\right)=(1,1)$ is a positive integer solution of the above equation. From

$$
2 X=c Y^{2} \pm(c+2)=c \pm(c+2),
$$

we get the solutions $(X, \pm Y)=(-1,1),(c+1,1)$ listed in Theorem 1.2. We will try to find the solutions of equation (3.3) satisfying $\left(x^{\prime}, \pm Y\right) \neq(1,1)$.

- If $2 \nmid c$, then the minimal solution of the equation

$$
(c+2) x^{2}-(c-2) y^{2}=4
$$

is $\left(x_{1}, y_{1}\right)=(1,1)$. We will apply Lemma 2.2 . As

$$
\left(\frac{\sqrt{c+2}+\sqrt{c-2}}{2}\right)^{3}=\frac{(c-1) \sqrt{c+2}+(c+1) \sqrt{c-2}}{2}
$$

we get $\left(x^{\prime}, Y\right)=(c-1, \sqrt{c+1})$, if $c+1$ is a square. Put $c+1=(2 s)^{2}, s \in \mathbb{N}$. Therefore, we have the solutions in the exceptional case (1.2) of Theorem 1.2, with $n$ even.

- If $4 \mid c$, then equation (3.3) becomes

$$
(c / 2+1) x^{\prime 2}-(c / 2-1) Y^{4}=2 .
$$


Using Lemma 2.3 and an argument similar to that of the above case, we obtain the solutions in the exceptional case (1.2) of Theorem 1.2, with $n$ odd.

- If $c \equiv 2(\bmod 4)$, then from equation $(3.3)$ we deduce

$$
((c / 2+1) / 2) x^{2}-((c / 2-1) / 2) Y^{4}=1
$$

or

$$
\left(c_{1}+1\right) x^{2}-c_{1} Y^{4}=1,
$$

where $c=4 c_{1}+2$. By Lemma 2.4, there is no solution satisfying $\left(x^{\prime}, \pm Y\right) \neq$ $(1,1)$.

3.3. The case $\mu=-c+2$. From the equation

$$
x^{2}-\left(c^{2}-4\right) y^{2}=4(-c+2),
$$

as in the case $\mu=c+2$, we can see that $(c-2) \mid x$. So, there exists $x^{\prime \prime} \in \mathbb{N}$ such that $x=(c-2) x^{\prime \prime}$. Then we have

$$
(c+2) y^{2}-(c-2) x^{\prime \prime 2}=4 .
$$

Now, since $y=Y^{2}$, the equation becomes

$$
(c+2) Y^{4}-(c-2) x^{\prime \prime 2}=4,
$$

where $x^{\prime \prime}=\frac{\left|2 X-c Y^{2}\right|}{c-2}$. The trivial solution $\left(x^{\prime \prime}, \pm Y\right)=(1,1)$ provides

$$
2 X=c Y^{2} \pm(c-2)=c \pm(c+2) .
$$

We obtain the solutions $(X, \pm Y)=(1,1),(c-1,1)$ listed in Theorem 1.2. Also, let us assume $\left(x^{\prime \prime}, \pm Y\right) \neq(1,1)$ in the following discussion.

- Case $2 \nmid c$ : We need to consider the equation

$$
(c+2) Y^{4}-(c-2) x^{\prime \prime 2}=4 .
$$

To apply Lemma 2.5 , we notice that $\left(x_{1}, y_{1}\right)=(1,1)$ is the minimal solution to

$$
(c+2) y^{2}-(c-2) x^{\prime \prime 2}=4 .
$$

Moreover, in general it is not true that $a x_{1}^{2}-3=x_{3}$. Using an argument similar to that of the case $\mu=c+2$ and $2 \nmid c$, we have $x_{3}=c-1$. So the additional solution comes from $a x_{1}^{2}-3=c-1$, where $c-1$ is a square. Put $c=$ $(2 s)^{2}+1, s \in \mathbb{N}$. Then we get the solution $\left(Y, x^{\prime \prime}\right)=\left(2 s,(2 s)^{2}+2\right)$ of equation (3.4). This is corresponding to the solutions $(X, \pm Y)=\left((2 s)^{4}+(2 s)^{2}-1,2 s\right)$ and $(X, \pm Y)=(1,2 s)$ seen in the case (1.2) of Theorem 1.2, with $n$ even.

- Case $4 \mid c$ : Equation (3.4) becomes

$$
(c / 2+1) Y^{4}-(c / 2-1) x^{\prime \prime 2}=2 .
$$

By Lemma 2.6, there is no additional solution.

- The most difficult case in the paper is $c \equiv 2(\bmod 4)$. From equation (3.4), we deduce

$$
((c / 2+1) / 2) Y^{4}-((c / 2-1) / 2) x^{\prime \prime 2}=1 .
$$


We cannot explicitly solve it. But we can use Lemma 2.7 to see that there is at most one non-trivial integer solution $\left(Y, x^{\prime \prime}\right)$. This will possibly provide at most two integer solutions to equation (1.1). This corresponds to case (1.2) of Theorem 1.2.

Finally, when $(c / 2+1) / 2=s^{2}+s+1$, by Lemma 2.8 , we obtain the case (1.2) of Theorem 1.2, with $n$ odd. This completes the proof of Theorem 1.2.

ACKNOWLEDGEMENTS.

The first author was supported by the Applied Basic Research Foundation of Sichuan Provincial Science-Technology Department (No. 2011JYZ032), and by NSF of Education Department of Sichuan Province (No. 13ZA0037, 13ZB0036), and by NSF of China (No. 11301363). The second author was supported in part, by OTKA grant K75566. The third author was supported in part, by the Hungarian Academy of Sciences, OTKA grants T67580, K75566, K100339, NK101680, NK104208, and the Project TÁMOP 4.2.1./B09/1/KONV-2010-0007 implemented through the New Hungary Development Plan co-financed by the European Social Fund and the European Regional Development Fund. The fourth author is grateful to Purdue University North Central for the support.

\section{REFERENCES}

[1] S. Akhtari, The Diophantine Equation $a X^{4}-b Y^{2}=1$, J. Reine Angew. Math. 630 (2009), 33-57.

[2] S. Akhtari, A. Togbé and P.G. Walsh, On the equation $a X^{4}-b Y^{2}=2$. Acta Arith. 131 (2008), 145-169.

[3] S. Akhtari, A. Togbé, and P.G. Walsh, Addendum on the equation $a X^{4}-b Y^{2}=2$, Acta Arith. 137 (2009), 199-202.

[4] M.A. Bennett, A. Togbé, and P.G. Walsh, A generalization of a theorem of Bumby on quartic Diophantine equations, Int. J. Number Theory, 2 (2006), 195-206.

[5] W. Bosma, J. Cannon and C. Playoust, The Magma algebra system. I. The user language, J. Symbolic Comput. 24 (1997), 235-265.

[6] J. H. E. Cohn, Twelve Diophantine equations, Arch. Math. 65 (1995), 130-133.

[7] T. W. Cusick, The Diophantine equation $x^{4}-k x^{2} y^{2}+y^{4}=1$, Arch. Math. 59 (1992), 345-347.

[8] A. Dujella, B. Ibrahimpašić and B. Jadrijević, Solving a family of quartic Thue inequalities using continued fractions, Rocky Mountain J. Math., 41 (2011), 11731182.

[9] B. He, O. Kihel and A. Togbé, Solutions of a class of quartic Thue inequalities, Computers and Mathematics with Applications 61 (2011), 2914-2923.

[10] C. Heuberger, Parametrized Thue equations: a survey, Proceedings of the RIMS symposium Analytic number theory and surrounding areas, Kyoto, Oct 18-22, 2004, RIMS Kôkyûroku 1511, August 2006, 82-91.

[11] D. H. Lehmer, An extended theory of Lucas' functions, Ann. of Math. (2) 31 (1930), 419-448.

[12] W. Ljunggren, Ein Satz über die Diophantische Gleichung $A x^{2}-B y^{4}=C(C=$ 1,2,4) Tolfte Skand. Matemheikerkongressen, Lund (1953), 188-194.

[13] W. Ljunggren. On the Diophantine equation $A x^{4}-B y^{2}=C(C=1,4)$. Math. Scand. 21 (1967), 149-158. A remark concerning the paper "On the Diophantine equation $A x^{4}-B y^{2}=C(C=1,4)$ ", Math. Scand. 22 (1968), 282. 
[14] F. Luca and P. G. Walsh, Squares in Lehmer sequences with Diophantine applications, Acta Arit. 100 (2001), 47-62.

[15] J. Luo and P. Yuan, Square-classes in Lehmer sequences having odd parameters and their applications, Acta Arith. 127 (2007), 49-62.

[16] M. Mignotte, A. Pethő and R. Roth, Complete solutions of a family of quartic Thue and index form equations, Math. Comp. 65 (1996), 341-354.

[17] A. Pethő, Complete solutions to families of quartic Thue equations, Math. Comp. $\mathbf{5 7}$ (1991), 777-798

[18] A. Togbé, P. M. Voutier and P. G. Walsh, Solving a family of Thue equations with an application to the equation $x^{2}-D y^{4}=1$, Acta Arith. 120 (2005), 39-58.

[19] P. M. Voutier, A further note on "On the equation $a X^{4}-b Y^{2}=2$ ", Acta Arith. 137 (2009), 203-206.

[20] I. Wakabayashi, On a family of quartic Thue inequations, J. Number Theory 66 (1997), 70-84.

[21] P. G. Walsh, A note on a theorem of Ljunggren and the Diophantine equations $x^{2}-$ $k x y^{2}+y^{4}=1,4$, Arch. Math. 73 (1999), 119-125.

[22] P. Yuan and Y. Li, Squares in Lehmer sequences and the Diophantine equation Ax ${ }^{4}$ $B y^{2}=2$, Acta Arith. 139 (2009), 275-302.

[23] P. Yuan and Z. Zhang, On the Diophantine Equation $a X^{4}-b Y^{2}=1$, Acta Math. Sinica (Chin. Ser.) 53 (2010), 443-454 (in Chinese).

B. $\mathrm{He}$

Department of Mathematics

ABa Teacher's College

Wenchuan, Sichuan 623000

P. R. China

E-mail: bhe@live.cn

I. Pink

Institute of Mathematics

P. O. Box 12, H-4010 Debrecen

Hungary

E-mail: pinki@science.unideb.hu

Á. Pintér

Institute of Mathematics, MTA-DE Research Group

"Equations, Functions and Curves"

Hungarian Academy of Sciences and University of Debrecen

P. O. Box 12, H-4010 Debrecen

Hungary

E-mail: apinter@science.unideb.hu

A. Togbé

Department of Mathematics

Purdue University North Central

1401 S. U.S. 421

Westville, IN 46391

USA

E-mail: atogbe@pnc.edu

Received: 3.2.2013.

Revised: 14.4 .2013 . \& 21.4.2013. 